\title{
Mathematical Calculation of Stray Losses in Transformer Tanks with a Stainless Steel Insert
}

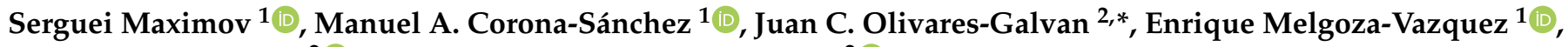 \\ Rafael Escarela-Perez ${ }^{2}\left(\mathbb{D}\right.$ and Victor $M$. Jimenez-Mondragon ${ }^{2}(\mathbb{D}$
}

1 Tecnológico Nacional de Mexico, Instituto Tecnológico de Morelia, PGIIE, Av. Tecnológico No. 1500, Lomas de Santiaguito, Morelia 58120, Mich., Mexico; sgmaximov@yahoo.com.mx (S.M.); manuel.cs@morelia.tecnm.mx (M.A.C.-S.); emv@ieee.org (E.M.-V.)

2 Departamento de Energía, Universidad Autónoma Metropolitana Azcapotzalco, Ciudad de México 02200, Mexico; r.escarela@ieee.org (R.E.-P.); vmjm@azc.uam.mx (V.M.J.-M.)

* Correspondence: jolivares@azc.uam.mx; Tel.: +52-135-1519-3760

Citation: Maximov, S;

Corona-Sánchez, M.A.; Olivares-Galvan, J.C.;

Melgoza-Vazquez, E.

Escarela-Perez, R.;

Jimenez-Mondragon, V.M.

Mathematical Calculation of Stray

Losses in Transformer Tanks with a Stainless Steel Insert. Mathematics 2021, 9, 184. https://doi.org/ $10.3390 /$ math 9020184

Received: 28 November 2020 Accepted: 13 January 2021 Published: 18 January 2021

Publisher's Note: MDPI stays neutral with regard to jurisdictional clai$\mathrm{ms}$ in published maps and institutional affiliations.

Copyright: $\odot 2021$ by the authors. Licensee MDPI, Basel, Switzerland. This article is an open access article distributed under the terms and conditions of the Creative Commons Attribution (CC BY) license (https:// creativecommons.org/licenses/by/ $4.0 /)$.

\begin{abstract}
At present it is claimed that all electrical energy systems operate with high values of efficiency and reliability. In electric power systems (EPS), electrical power and distribution transformers are responsible for transferring the electrical energy from power stations up to the load centers. Consequently, it is mandatory to design transformers that possess the highest efficiency and reliability possible. Considerable power losses and hotspots may exist in the bushing region of a transformer, where conductors pass through the tank. Most transformer tanks are made of low-carbon steel, for economical reasons, causing the induction of high eddy currents in the bushing regions. Using a non-magnetic insert in the transformer tank can reduce the eddy currents in the region and as a consequence avoid overheating. In this work, analytical formulations were developed to calculate the magnetic field distribution and the stray losses in the transformer region where bushings are mounted, considering a stainless steel insert (SSI) in the transformer tank. Previously, this problem had only been tackled with numerical models. Several cases were analyzed considering different non-magnetic insert sizes. Additionally, a numerical study using a two dimensional (2D) finite element (FE) axisymmetric model was carried out in order to validate the analytical results. The solved cases show a great concordance between models, obtaining relative errors between the solutions of less than two percent.
\end{abstract}

Keywords: power transformer; stray losses; analytical methods; finite element method

\section{Introduction}

Nowadays, electric power systems (EPS) are constantly changing. The use of new technologies such as smart-grids, micro-grids and renewable energy systems demand high flexibility, performance and efficiency in the EPS. The transformer is a fundamental component in these systems, which is present in different stages of the EPS, such as at the generation, transmission and distribution stages [1,2]. Losses in transformers appear in their different components, such as: windings, insulation, core and tank. These losses depend on the operating conditions of the transformer (nominal values, DC bias, presence of harmonics, etc.) and the electrical and magnetic properties of the materials. The study of transformer losses is an active area of investigation because there is a compromise between design and cost. Power losses in transformers can be separated into two types: no-load and load losses; the no-load losses originate in the transformer core and the load losses are composed by the ohmic losses in the windings and the stray losses. As the rated power increases in transformers, the stray losses increase significantly. The stray losses in structural components, such as the tank, decrement the efficiency of the transformer considerably [3]. Losses in structural components in power transformers are due to stray fluxes; when a time varying flux impinges on a conductive element it induces a current in 
it, generating Joule losses. Besides power losses, hotspots in structural components caused by this phenomenon may appear. For these reasons, there are diverse techniques to reduce losses without unduly increasing the final cost. A crucial structural part of a transformer is the tank region where the bushings are mounted. The field concentration and overheating in this zone can cause damage to the device [4].

Analytical and numerical models can be employed for the design of the transformer taking into account the well-known advantages of each one: analytic models provide accuracy, less calculation time and simplicity once the model has been developed, whereas numeric models allow one to solve complex geometries and deal with nonlinear materials. Several research works, analyzing the magnetic field distribution and the eddy current power losses in the bushing transformer region, using either numerical or analytical models, have been carried out previously [1,4-15].

An analytical model is proposed in $[4,5]$ to determine the power losses based on Poynting's theorem. To obtain the solution some semi-empirical coefficients are required. Analytical expressions were developed in [1] to calculate the magnetic field and the stray losses in the transformer tank near the bushing. The configuration is modeled with a finite disk and a conductor in the center. It is considered that the axial component of the induced current density has only a small contribution to the power losses, and therefore it is disregarded. This automatically implies that the solution obtained will be an approximation. A study to reduce stray losses in a pad mounted transformer wall using an insert plate is presented in [6,7]. These studies were carried out using two dimensional (2D) and three dimensional (3D) finite element (FE) analysis. The results were validated with experimental tests in different combinations. Additionally, numerical results were compared with empirical formulas. In these works, it was verified that the use of non-magnetic inserts in the transformer tank reduce eddy current losses. A transient analysis was carried out in [8] to compute the power losses in the low-carbon steel tank of a current transformer, taking into account different insert configurations and materials. The analyses were done using a 3D FE model. It was also concluded that using non-magnetic inserts reduce power losses in the tank. In $[9,11]$ the temperature distribution on transformer covers is considered. The stray losses in the tank were analytically calculated using Turowski's formula [4]. Maximov et al. [10] presented a study of eddy current losses in the tank of a transformer. Numerical results and analytical formulas were obtained for the losses as a function of the current. However, in the solution to this problem only two materials were considered: the tank and the air. In $[12,13]$, the determination of eddy current losses and temperature distribution in the zone of the transformers bushing are presented. The study was carried out with the finite difference method. An analytical solution for the bushing regions, using the same model geometry of [10], is proposed in [14]. However in this research the presence of harmonics in the current is considered. Oliveira et al. [15] developed a time domain model to determine the eddy currents in the transformer tank walls considering different types of excitations. The results obtained with the proposed model were validated with a $3 \mathrm{D}$ FE solution. The tank wall was considered to be made of a single magnetic material.

As can be appreciated from the literature reviewed above, in previous research where a stainless steel insert (SSI) is added to reduce the power losses in the tank, a numerical model is employed in the analysis, whereas analytical models are developed only for the cases where the tank in the bushing region is made from a single material. In this paper a mathematical model is proposed to determine the magnetic field distribution in the tank wall bushing regions, considering a tank wall composed of two different materials, a stainless steel section representing the insert and a low-carbon steel section modeling the rest of the tank. An analytical calculation is also developed to determine the stray losses in the bushing region.

\section{Model}

The bushing transformer region is considered through an idealized model. Consider an infinite conductor that passes the transformer tank wall at a right angle across a circular 
hole. The tank is considered to have an annular shape with thickness $h$ and inner and outer radii $c$ and $b$, respectively. A SSI is also considered in the tank, with a radial length $c-a$. Figure 1 shows the geometry of the model. The analysis domain is divided into four regions: $\Omega_{1}$ is the region existing between the conductor and the tank wall, namely, the circular hole; $\Omega_{1}$ is defined by $r_{0} \leq r \leq a,-h / 2 \leq z \leq h / 2$, where $r_{0}$ is the radius of the conductor. Region $\Omega_{2}$ represents the carbon steel tank wall, that is, $c \leq r \leq b$, $-h / 2 \leq z \leq h / 2$. Region $\Omega_{3}$ is the SSI, $a \leq r \leq c,-h / 2 \leq z \leq h / 2$. Finally, region $\Omega_{4}$ is the medium at both sides of the tank wall, considered as air, $r \geq r_{0},|z|>h / 2$.

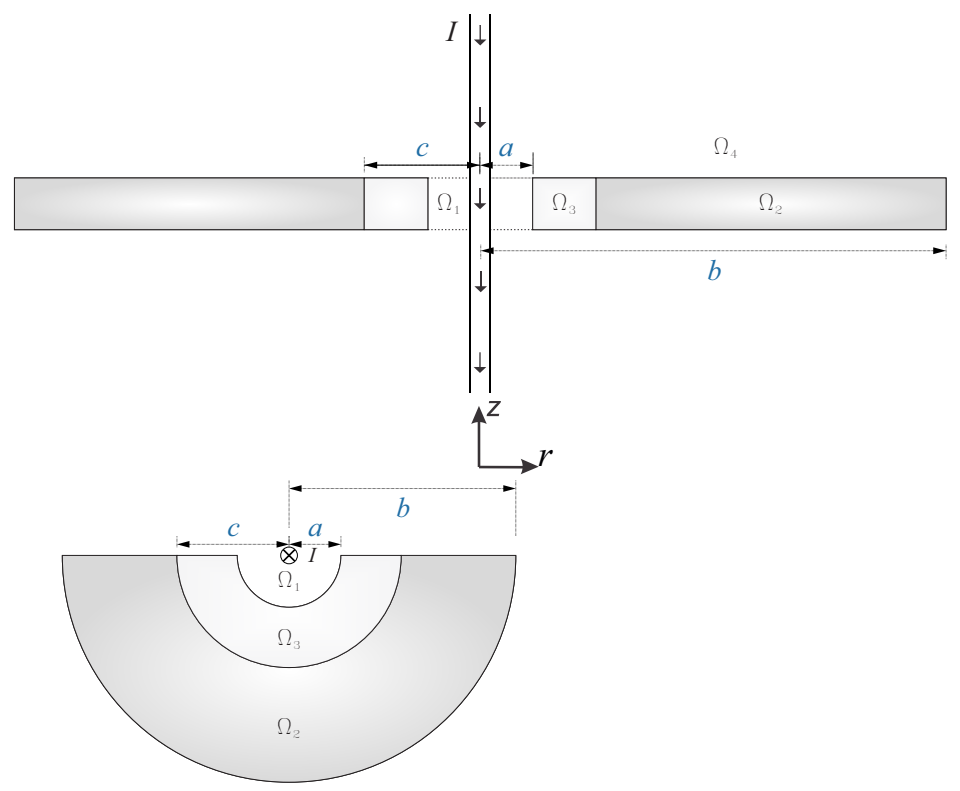

Figure 1. Geometry and parameters of the model.

A conductor passing through the hole transports alternating electric current of the form:

$$
I(t)=I e^{j \omega t},
$$

where $I$ is the current amplitude. Maxwell's equations in the frequency domain, in each region $\Omega_{k}$, have the following form:

$$
\begin{array}{ll}
\nabla \times \mathbf{E}_{k}=-j \omega \mu_{0} \mu_{k} \mathbf{H}_{k}, & \nabla \cdot \mathbf{H}_{k}=0, \\
\nabla \times \mathbf{H}_{k}=\sigma_{k} \mathbf{E}_{k}, & \nabla \cdot \mathbf{E}_{k}=0,
\end{array}
$$

where $k=1$ corresponds to air and the hole (region $\left.\Omega_{1}\right), k=2$ corresponds to the carbon steel (region $\Omega_{2}$ ) and $k=3$ is associated with the stainless insert (region $\Omega_{3}$ ). Additionally, $\sigma_{1}=0$ and $\mu_{1}=\mu_{3}=1$. Because of the axial symmetry of the system and the symmetry with respect to the plane $z=0$, the solution to the system of Equations (1) can be sought as follows:

$$
\mathbf{H}_{k}=H_{k \varphi}(r, z) \mathbf{e}_{\varphi}, \quad \mathbf{E}_{k}=E_{k r}(r, z) \mathbf{e}_{r}+E_{k \varphi}(r, z) \mathbf{e}_{\varphi},
$$

where $H_{k \varphi}(r, z)$ is an even function with respect to the variable $z$; i.e.,

$$
H_{k \varphi}(r,-z)=H_{k \varphi}(r, z) .
$$
hole is:

Due to Ampère's circuital law and axial symmetry, the magnetic field in air and the

$$
H_{1 \varphi}(r)=\frac{I}{2 \pi r}
$$


Boundary conditions between regions $\Omega_{1}$ and $\Omega_{2,3}$ after taking into account Equation (4) assume the following form (see $[10,14])$ :

$$
\begin{array}{ll}
\left.H_{3 \varphi}\right|_{r=a}=\frac{I}{2 \pi a}, & \left.H_{2 \varphi}\right|_{r=b}=\frac{I}{2 \pi b}, \\
\left.\frac{1}{r} \frac{\partial\left(r H_{3 \varphi}\right)}{\partial r}\right|_{z=h / 2}=0, & \left.\frac{1}{r} \frac{\partial\left(r H_{2 \varphi}\right)}{\partial r}\right|_{z=h / 2}=0, \\
\left.H_{3 \varphi}\right|_{z=h / 2}=\frac{I}{2 \pi r}, & \left.H_{2 \varphi}\right|_{z=h / 2}=\frac{I}{2 \pi r} .
\end{array}
$$

Additionally, on the boundary that separates regions $\Omega_{2}$ and $\Omega_{3}$ we have:

$$
\begin{array}{r}
\left.H_{3 \varphi}\right|_{r=c}=\left.H_{2 \varphi}\right|_{r=c^{\prime}} \\
\left.\frac{1}{\sigma_{3}} \frac{1}{r} \frac{\partial\left(r H_{3 \varphi}\right)}{\partial r}\right|_{r=c}=\left.\frac{1}{\sigma_{2}} \frac{1}{r} \frac{\partial\left(r H_{2 \varphi}\right)}{\partial r}\right|_{r=c} ^{,},
\end{array}
$$

\section{Analytical Solution}

Maxwell's equations in regions $\Omega_{2}$ and $\Omega_{3}$ reduce to:

$$
\frac{1}{r} \frac{\partial}{\partial r}\left(r \frac{\partial H_{k \varphi}}{\partial r}\right)+\frac{\partial^{2} H_{k \varphi}}{\partial z^{2}}-\frac{H_{k \varphi}}{r^{2}}-j \omega \sigma_{k} \mu_{0} \mu_{k} H_{k \varphi}=0 .
$$

Equation (8) has been previously solved $[10,14]$. The solution of this equation has the following form [14]:

$$
\begin{aligned}
H_{k \varphi}(r, z) & =\left(\frac{A_{k}}{r}+B_{k} r\right) \cosh \left(\beta_{k} z\right) \\
& +\sum_{n=0}^{\infty}\left\{C_{k, n} I_{1}\left(\lambda_{k, n} r\right)+D_{k, n} K_{1}\left(\lambda_{k, n} r\right)\right\} \cos \left(\varkappa_{n} z\right),
\end{aligned}
$$

where $I_{1}\left(\lambda_{k, n} r\right)$ and $K_{1}\left(\lambda_{k, n} r\right)$ are the modified Bessel functions of the first order,

$$
\begin{aligned}
\beta_{k}^{2} & =j \omega \sigma_{k} \mu_{0} \mu_{k}, \quad \varkappa_{n}=\frac{(2 n+1) \pi}{h} \\
\lambda_{k, n}^{2} & =\varkappa_{n}^{2}+\beta_{k}^{2} \quad k=2,3, \quad n \in \mathbb{Z}^{+} .
\end{aligned}
$$

Constants $A_{k}, B_{k}, C_{k, n}$ and $D_{k, n}$ are to be obtained from boundary conditions (5)-(7). Substitution of solution (9) into the last boundary conditions of (5) yields:

$$
B_{k}=0, \quad A_{k}=\frac{I}{2 \pi \cosh \left(\beta_{k} h / 2\right)} .
$$

As a result,

$$
\begin{aligned}
H_{k \varphi}(r, z) & =\frac{I}{2 \pi r} \frac{\cosh \left(\beta_{k} z\right)}{\cosh \left(\beta_{k} h / 2\right)} \\
& +\sum_{n=0}^{\infty}\left\{C_{k, n} I_{1}\left(\lambda_{k, n} r\right)+D_{k, n} K_{1}\left(\lambda_{k, n} r\right)\right\} \cos \left(\varkappa_{n} z\right),
\end{aligned}
$$

The convergence of the generalized Fourier-series (10) is provided by the general theory of linear partial differential equations, with Hermitian differential operators and 
boundary conditions (5)-(7). However, the convergence can be also proved explicitly, which has been done, for instance, in the Appendix section of [10].

At the same time, since function $\cosh \left(\beta_{k} z\right) / \cosh \left(\beta_{k} h / 2\right)$ can be expanded in a Fourier series as follows [10]:

$$
\frac{\cosh \left(\beta_{k} z\right)}{\cosh \left(\beta_{k} h / 2\right)}=\sum_{n=0}^{\infty} \frac{4(-1)^{n} \varkappa_{n}}{\lambda_{k, n}^{2} h} \cos \left(\varkappa_{n} z\right),
$$

then, Equation (10) takes the form:

$$
\begin{aligned}
H_{k \varphi}(r, z) & =\sum_{n=0}^{\infty}\left\{C_{k, n} I_{1}\left(\lambda_{k, n} r\right)+D_{k, n} K_{1}\left(\lambda_{k, n} r\right)\right. \\
& \left.+\frac{2 I}{\pi r} \frac{(-1)^{n} \varkappa_{n}}{\lambda_{k, n}^{2} h}\right\} \cos \left(\varkappa_{n} z\right),
\end{aligned}
$$

Let us substitute general solution (11) for $H_{3 \varphi}(r, z)$ into boundary condition $\left.H_{3 \varphi}\right|_{r=a}=$ $I / 2 \pi a$. We obtain within the interval $-h / 2 \leq z \leq h / 2$ :

$$
\sum_{n=0}^{\infty}\left\{C_{3, n} I_{1}\left(\lambda_{3, n} a\right)+D_{3, n} K_{1}\left(\lambda_{3, n} a\right)+\frac{2 I}{\pi a} \frac{(-1)^{n} \varkappa_{n}}{\lambda_{3, n}^{2} h}\right\} \cos \left(\varkappa_{n} z\right)=\frac{I}{2 \pi a} .
$$

On the other hand, within the same interval, we can write (see [10]):

$$
\sum_{n=0}^{\infty} \frac{4(-1)^{n}}{\varkappa_{n} h} \cos \left(\varkappa_{n} z\right)=1
$$

After substituting this result into (12) we come to the following equation:

$$
\begin{aligned}
\sum_{n=0}^{\infty}\left\{C_{3, n} I_{1}\left(\lambda_{3, n} a\right)+\right. & D_{3, n} K_{1}\left(\lambda_{3, n} a\right) \\
& \left.+\frac{2 I}{\pi a} \frac{(-1)^{n} \varkappa_{n}}{\lambda_{3, n}^{2} h}\right\} \cos \left(\varkappa_{n} z\right)=\sum_{n=0}^{\infty} \frac{2 I}{\pi a} \frac{(-1)^{n}}{\varkappa_{n} h} \cos \left(\varkappa_{n} z\right),
\end{aligned}
$$

which, in turn, leads to the following:

$$
C_{3, n} I_{1}\left(\lambda_{3, n} a\right)+D_{3, n} K_{1}\left(\lambda_{3, n} a\right)+\frac{2 I}{\pi a} \frac{(-1)^{n} \varkappa_{n}}{\lambda_{3, n}^{2} h}=\frac{2 I}{\pi a} \frac{(-1)^{n}}{\varkappa_{n} h} .
$$

This equation, after some simple algebraic operations, becomes:

$$
C_{3, n} I_{1}\left(\lambda_{3, n} a\right)+D_{3, n} K_{1}\left(\lambda_{3, n} a\right)=\frac{2 I}{\pi a} \frac{(-1)^{n} \beta_{3}^{2}}{\lambda_{3, n}^{2} \varkappa_{n} h} .
$$

A similar result can be obtained from boundary condition $\left.H_{2 \varphi}\right|_{r=b}=I / 2 \pi b$ :

$$
C_{2, n} I_{1}\left(\lambda_{2, n} b\right)+D_{2, n} K_{1}\left(\lambda_{2, n} b\right)=\frac{2 I}{\pi b} \frac{(-1)^{n} \beta_{2}^{2}}{\lambda_{2, n}^{2} \varkappa_{n} h}
$$


Boundary conditions (6) and (7) result in the following equations:

$$
\begin{gathered}
C_{2, n} I_{1}\left(\lambda_{2, n} c\right)+D_{2, n} K_{1}\left(\lambda_{2, n} c\right)+\frac{2 I}{\pi c} \frac{(-1)^{n} \varkappa_{n}}{\lambda_{2, n}^{2} h} \\
=C_{3, n} I_{1}\left(\lambda_{3, n} c\right)+D_{3, n} K_{1}\left(\lambda_{3, n} c\right)+\frac{2 I}{\pi c} \frac{(-1)^{n} \varkappa_{n}}{\lambda_{3, n}^{2} h} \\
\frac{\lambda_{2, n}}{\sigma_{2}}\left\{C_{2, n} I_{0}\left(\lambda_{2, n} c\right)-D_{2, n} K_{0}\left(\lambda_{2, n} c\right)\right\} \\
=\frac{\lambda_{3, n}}{\sigma_{3}}\left\{C_{3, n} I_{0}\left(\lambda_{3, n} c\right)-D_{3, n} K_{0}\left(\lambda_{3, n} c\right)\right\}
\end{gathered}
$$

The system of linear Equations (14)-(17), with respect to the constants $C_{k, n}$ and $D_{k, n}$, $k=\overline{1, n}$, is easy to solve. However, there is no necessity to solve this system of equations exactly, since the magnetic field rapidly decays for an increasing outer radius $b$. This principle can be formally taken into account in the system of Equations (14)-(17) by considering radius $b$ to be sufficiently high (formally, $b \rightarrow \infty$ ). The validity of this assumption for the calculation of transformer tanks losses has been shown previously in $[10,14]$. By applying the limit to Equation (15) when $b \rightarrow \infty$ and taking into account the asymptotic behavior of the modified Bessel functions $I_{1}(x)$ and $K_{1}(x)$, namely,

$$
\begin{aligned}
I_{1}(x) & \propto \frac{e^{x}}{\sqrt{2 \pi x}}\left(1+\mathcal{O}\left(x^{-1}\right)\right), \\
K_{1}(x) & \propto \sqrt{\frac{\pi}{2 x}} e^{-x}\left(1+\mathcal{O}\left(x^{-1}\right)\right),
\end{aligned}
$$

it follows that $C_{2, n}=0$. Through substitution of the result into Equations (14)-(17) and solving this system of equations with respect to $C_{3, n}, D_{3, n}$ and $D_{2, n}$, we obtain:

$$
\begin{array}{r}
C_{3, n}=\frac{2 I}{\pi \Delta} \frac{(-1)^{n}}{\lambda_{3, n}^{2} h}\left\{\frac{\beta_{3}^{2}}{\varkappa_{n} a}\left[\frac{\lambda_{2, n}}{\sigma_{2}} K_{0}\left(\lambda_{2, n} c\right) K_{1}\left(\lambda_{3, n} c\right)-\frac{\lambda_{3, n}}{\sigma_{3}} K_{1}\left(\lambda_{2, n} c\right) K_{0}\left(\lambda_{3, n} c\right)\right]\right. \\
\left.-\frac{\varkappa_{n}\left(\beta_{3}^{2}-\beta_{2}^{2}\right)}{\lambda_{2, n} \sigma_{2} c} K_{0}\left(\lambda_{2, n} c\right) K_{1}\left(\lambda_{3, n} a\right)\right\}, \\
D_{3, n}=-\frac{2 I}{\pi \Delta} \frac{(-1)^{n}}{\lambda_{3, n}^{2} h}\left\{\frac{\beta_{3}^{2}}{\varkappa_{n} a}\left[\frac{\lambda_{2, n}}{\sigma_{2}} K_{0}\left(\lambda_{2, n} c\right) I_{1}\left(\lambda_{3, n} c\right)+\frac{\lambda_{3, n}}{\sigma_{3}} K_{1}\left(\lambda_{2, n} c\right) I_{0}\left(\lambda_{3, n} c\right)\right]\right. \\
\left.-\frac{\varkappa_{n}\left(\beta_{3}^{2}-\beta_{2}^{2}\right)}{\lambda_{2, n} \sigma_{2} c} K_{0}\left(\lambda_{2, n} c\right) I_{1}\left(\lambda_{3, n} a\right)\right\}
\end{array}
$$

and

$$
D_{2, n}=\frac{2 I}{\pi \Delta c} \frac{(-1)^{n}}{\lambda_{3, n} \sigma_{3} h}\left\{\varkappa_{n} \frac{\beta_{3}^{2}-\beta_{2}^{2}}{\lambda_{2, n}^{2}}\left[K_{1}\left(\lambda_{3, n} a\right) I_{0}\left(\lambda_{3, n} c\right)+I_{1}\left(\lambda_{3, n} a\right) K_{0}\left(\lambda_{3, n} c\right)\right]-\frac{\beta_{3}^{2}}{\varkappa_{n} \lambda_{3, n} a}\right\}
$$

where

$$
\begin{aligned}
\Delta=\left[\frac{\lambda_{2, n}}{\sigma_{2}} K_{0}\left(\lambda_{2, n} c\right) K_{1}\left(\lambda_{3, n} c\right)-\frac{\lambda_{3, n}}{\sigma_{3}} K_{1}\left(\lambda_{2, n} c\right) K_{0}\left(\lambda_{3, n} c\right)\right] I_{1}\left(\lambda_{3, n} a\right) \\
-\left[\frac{\lambda_{2, n}}{\sigma_{2}} K_{0}\left(\lambda_{2, n} c\right) I_{1}\left(\lambda_{3, n} c\right)+\frac{\lambda_{3, n}}{\sigma_{3}} K_{1}\left(\lambda_{2, n} c\right) I_{0}\left(\lambda_{3, n} c\right)\right] K_{1}\left(\lambda_{3, n} a\right) .
\end{aligned}
$$


Then, constant $C_{2, n}$ can be approximately calculated from Equation (15) as follows:

$$
C_{2, n}=\frac{1}{I_{1}\left(\lambda_{2, n} b\right)}\left\{\frac{2 I}{\pi b} \frac{(-1)^{n} \beta_{2}^{2}}{\lambda_{2, n}^{2} \varkappa_{n} h}-D_{2, n} K_{1}\left(\lambda_{2, n} b\right)\right\} .
$$

Solution (10) is an infinite sum that can be truncated at a term with number $N-1$ and by introducing the Lanczos sigma factor (see [16,17]):

$$
\varsigma_{n}=\frac{\sin (\pi n / N)}{\pi n / N}
$$

to suppress Gibbs' oscillations. Then, solution (11) takes the following form:

$$
\begin{aligned}
H_{k \varphi}(r, z) & =\frac{I}{2 \pi r} \frac{\cosh \left(\beta_{k} z\right)}{\cosh \left(\beta_{k} h / 2\right)} \\
& +\sum_{n=0}^{N-1} \varsigma_{n}\left\{C_{k, n} I_{1}\left(\lambda_{k, n} r\right)+D_{k, n} K_{1}\left(\lambda_{k, n} r\right)\right\} \cos \left(\varkappa_{n} z\right),
\end{aligned}
$$

\section{Electric Field and Eddy Current Losses}

Eddy current losses in the transformer tank wall have an ohmic nature. The averaged power loss density over a period is as follows [10,14]:

$$
P(r, z)=\frac{1}{T} \int_{0}^{T} \sigma(r, z)|\mathbf{E}(r, z, t)|^{2} d t=\frac{|\mathbf{j}(r, z)|^{2}}{2 \sigma(r, z)}
$$

where $T=2 \pi / \omega$ is the period. The tank wall conductivity $\sigma(r, z)$ is a function of the coordinates due to the insert in the tank wall, and $\mathbf{j}(r, z)$ is the current density in the frequency domain. Then, the total losses in the tank wall are [10]:

$$
\begin{aligned}
P_{\text {tot }}= & \int_{0}^{2 \pi} d \varphi \int_{a}^{b} r d r \int_{-h / 2}^{h / 2} d z P(r, z)=\pi \int_{a}^{b} r d r \int_{-h / 2}^{h / 2} d z \frac{|\mathbf{j}(r, z)|^{2}}{\sigma(r, z)} \\
& =\frac{\pi}{\sigma_{3}} \int_{a}^{c} r d r \int_{-h / 2}^{h / 2} d z\left(\left|j_{3, r}(r, z)\right|^{2}+\left|j_{3, z}(r, z)\right|^{2}\right) \\
& +\frac{\pi}{\sigma_{2}} \int_{c}^{b} r d r \int_{-h / 2}^{h / 2} d z\left(\left|j_{2, r}(r, z)\right|^{2}+\left|j_{2, z}(r, z)\right|^{2}\right) .
\end{aligned}
$$

The current density can be obtained from Maxwell's Equations (1) as follows:

$$
\mathbf{j}_{k}=\nabla \times \mathbf{H}_{k}=j_{k, r}(r, z) \mathbf{e}_{r}+j_{k, z}(r, z) \mathbf{e}_{z}
$$

where

$$
\begin{aligned}
j_{k, r}(r, z) & =-\frac{I \beta_{k}}{2 \pi r} \frac{\sinh \left(\beta_{k} z\right)}{\cosh \left(\beta_{k} h / 2\right)} \\
& +\sum_{n=0}^{N-1} \varsigma_{n} \varkappa_{n}\left\{C_{k, n} I_{1}\left(\lambda_{k, n} r\right)+D_{k, n} K_{1}\left(\lambda_{k, n} r\right)\right\} \sin \left(\varkappa_{n} z\right)
\end{aligned}
$$

and

$$
j_{k, z}(r, z)=\sum_{n=0}^{N-1} \varsigma_{n} \lambda_{k, n}\left\{C_{k, n} I_{0}\left(\lambda_{k, n} r\right)-D_{k, n} K_{0}\left(\lambda_{k, n} r\right)\right\} \cos \left(\varkappa_{n} z\right)
$$




\section{Study Cases and Discussion}

Several study cases are carried out in this section with different SSI sizes in the tank wall. In order to compare the results obtained with our analytical formulas, the solution in each case is also computed with a 2D FE model [18]. The low-carbon steel has the following properties: a relative permeability $\mu_{r}=100$, a conductivity $\sigma=7.0 \times 10^{6} \mathrm{~S} / \mathrm{m}$ and a relative permittivity $\epsilon_{r}=1$. The SSI has a relative permeability $\mu_{r}=1.0$, a conductivity $\sigma=1.1 \times 10^{6} \mathrm{~S} / \mathrm{m}$ and a relative permittivity $\epsilon_{r}=1$. The model dimensions in all the cases are $a=8.5 \mathrm{~cm}, b=34 \mathrm{~cm}$ and $h=12.7 \mathrm{~mm}$. The current $I$ carried by the conductor is $5000 \mathrm{~A}$ at frequency $f=60 \mathrm{~Hz}$. Various radial distances of the insert $(c-a)$ are considered, which are obtained by varying the percentage of the tank wall volume that corresponds to the SSI. This means that if the insert volume is $0 \%$, the tank wall is made only of low-carbon steel and therefore $c=a$. On other hand, if the insert volume is $100 \%$, the tank wall would be made exclusively of the stainless steel and its radial distance would be given by $c-a=b-a=255 \mathrm{~mm}$.

The numerical solution is obtained with a time-harmonic $2 \mathrm{D}$ eddy current axisymmetric FE model. A special 2D formulation is applied considering that field configuration is such that the magnetic field has only one component normal to the plane and the current density has its components in the plane, which matches with our problem. This formulation is incorporated into our FE code FLD and has been compared with analytical and 3D FE solutions, obtaining great accuracy in all cases [18]. FLD is a set of computer programs and routines, developed by the authors, for the analysis of electromagnetic problems using the FE method, which is programmed in Fortran 95 [19]. Using a 2D FE model permits a large number of simulations in a much shorter time and without the computational cost of a 3D model. Moreover, the geometry of the proposed model is represented faithfully by a 2D axisymmetric model.

The analysis domain considered in the FE axisymmetric model is composed by regions $\Omega_{3}$ and $\Omega_{4}$. A Dirichlet boundary condition, obtained by applying Ampère's circuital law (4), is assigned to nodes located at the periphery of the model. Figure 2 shows the FE meshes used to solve two different cases. Figure 2a is the mesh used for the case where the tank wall of the transformer lacks an SSI. Figure $2 \mathrm{~b}$ shows a case where an insert exists, modeled by the left blue region. The mesh used for each case results from an automatic mesh adaptation procedure; regions with a rapid variation of the field, after the iterative procedure, will contain a higher density of elements. In all cases second order elements were employed. Details of the implementation of the automatic mesh adaptation are reported in [18]. In both cases, most of the elements are in the periphery of the region $\Omega_{2}$, consisting of low-carbon steel, due to the skin effect in this material.

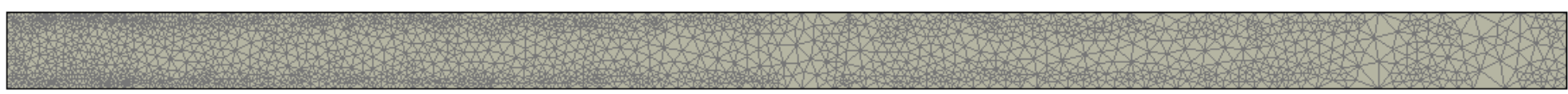

(a)

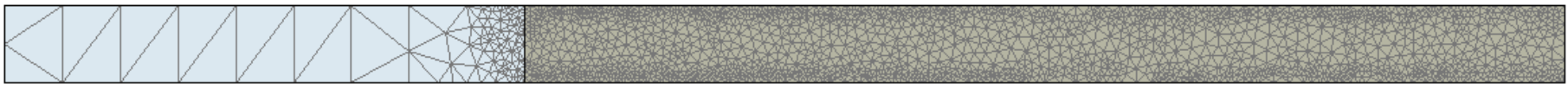

(b)

Figure 2. Mesh of the 2D FE axisymmetric model. (a) Tank wall without insert, case $c=a$. (b) Tank wall considering an insert, case $c-a=85.0 \mathrm{~mm}$.

In Figure 3 the magnetic field distribution of $H_{\varphi}(r, z)$, obtained with (4) and (24), is presented for different radii of the SSI. Observe how the magnetic field penetrates the tank wall according with the SSI size. Since the low-carbon steel possesses greater permeability and conductivity than the stainless steel, it has a smaller depth of penetration $(\delta)$. Hence, the magnetic field decays rapidly in region $\Omega_{2}$, whereas the magnetic field penetrates easily to region $\Omega_{3}$. 


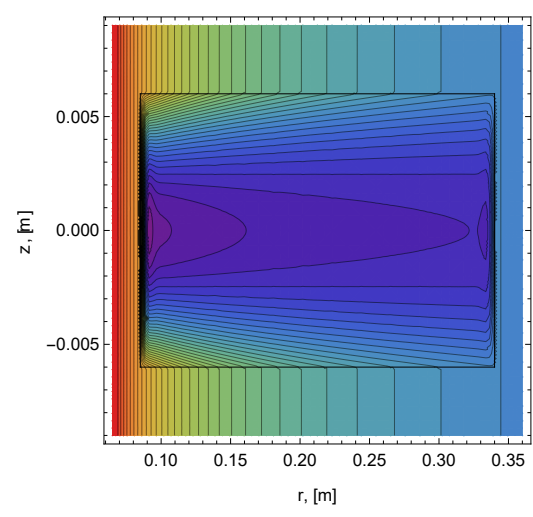

(a)

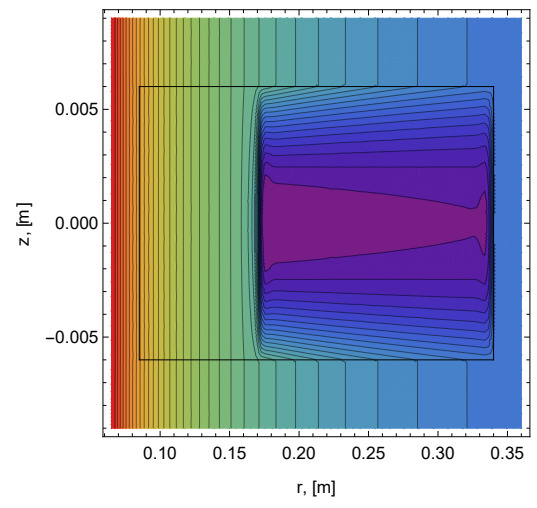

(c)

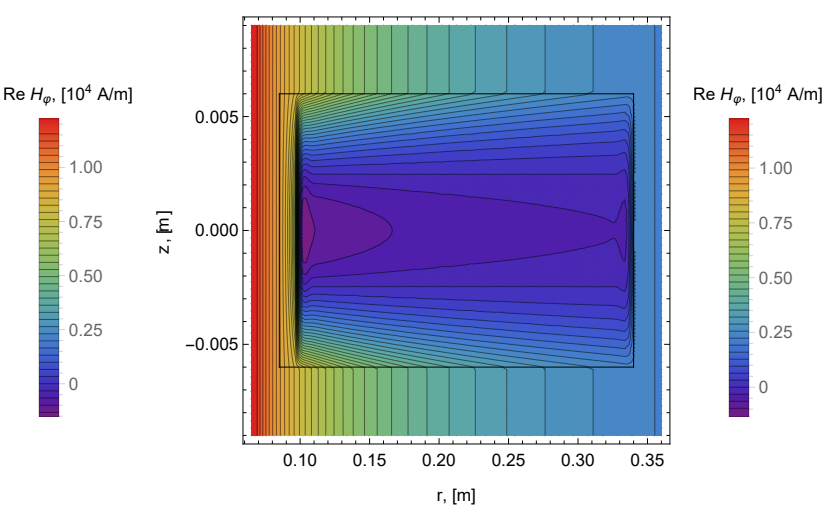

(b)

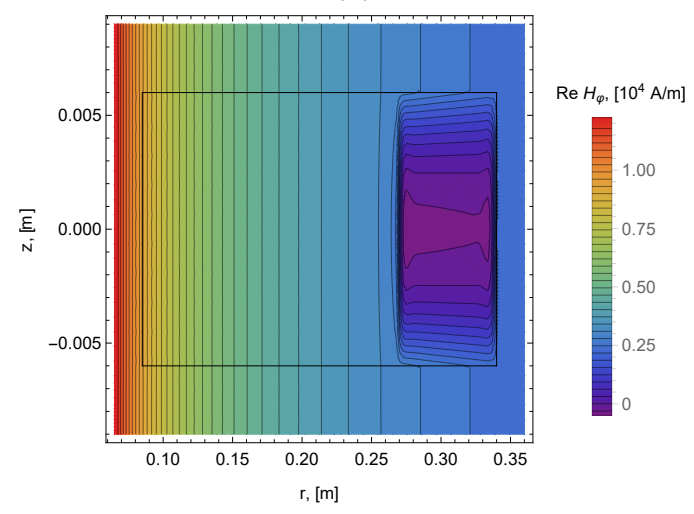

(d)

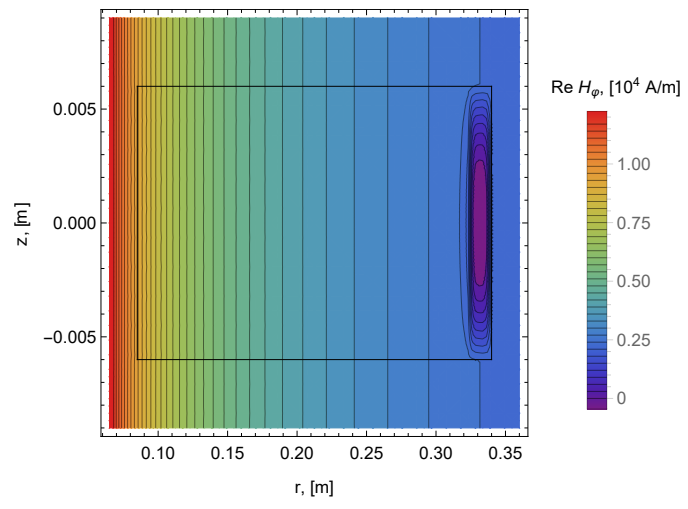

(e)

Figure 3. Magnetic field distribution inside the tank wall. (a) Case $c=a$. (b) Case $c-a=11.914 \mathrm{~mm}$. (c) Case $c-a=85 \mathrm{~mm}$. (d) Case $c-a=183.89 \mathrm{~mm}$. (e) Case $c-a=238.67 \mathrm{~mm}$.

Figure 4 shows two cases of the magnetic field penetration in the tank wall and the eddy current density obtained with 2D FE simulation. Figure $4 \mathrm{a}$ is the case where there is no insert. In this solution the skin effect phenomena in the tank wall can also be seen, producing the greater magnitude of $H_{\varphi}$ in the inner radius, the nearest region to the conductor. Figure $4 \mathrm{~b}$ presents the case in which the radial distance of the stainless insert is $c-a=85 \mathrm{~mm}$. In this case, the closed path of the eddy currents in the tank can be seen. The magnitude of the eddy current density is greater in the low-carbon steel. 


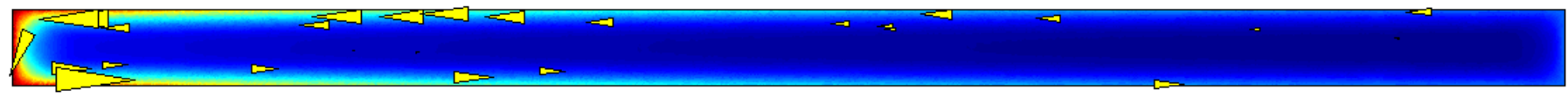

(a)

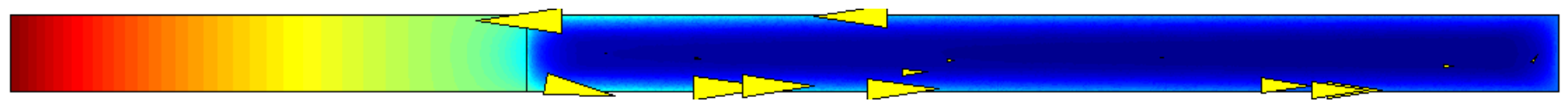

(b)

Figure 4. Magnitude of $H_{\varphi}$ (color) and eddy current density (arrows) in the tank wall. (a) Case $c=a$. (b) Case $c-a=85.0 \mathrm{~mm}$.

Figure 5 shows the magnetic field $H_{\varphi}$ evaluated at the center of the tank wall $(z=0)$ for different insert radii. For all cases, the analytical solution, developed in this work and the numerical one obtained with the 2D FE model are compared. It can be seen that for all the cases, the values of $H_{\varphi}$ calculated analytically match very closely the values obtained numerically, demonstrating the validity of the analytical formula (24). In these graphs, the behavior of the magnetic field inside the tank wall can be noticed more clearly: when $H_{\varphi}$ penetrates in the low-carbon steel, it decreases rapidly, having a greater variation at the edges of the tank. The magnetic field outside of the tank wall decreases according to the distance to the conductor, as established in (4). Table 1 shows the relative error between the analytical solution and numerical solutions calculated for these cases. The maximum relative error obtained is $1.71 \%$, which demonstrates the validity of the solutions.

Table 1. Relative error between solutions.

\begin{tabular}{|c|c|}
\hline $\begin{array}{l}\text { Case } \\
c-a \\
(\mathrm{~mm})\end{array}$ & $\begin{array}{c}\text { Relative } \\
\text { Error } \\
(\%)\end{array}$ \\
\hline 0 & 0.52 \\
\hline 11.914 & 1.37 \\
\hline 85 & 0.83 \\
\hline 183.79 & 1.71 \\
\hline 238.67 & 0.45 \\
\hline
\end{tabular}

The relative error is calculated with

$$
\text { relative error }=\frac{\max _{i=\overline{1, n}}\left|f_{i}-g_{i}\right|}{\max _{i=\overline{1, n}}\left|g_{i}\right|} 100 \%
$$

where $f_{i}$ and $g_{i}$ are the numerical and analytical solutions respectively, evaluated at point $i$, while $n$ is the total number of points considered.

The eddy current losses $P_{e}$ in the bushing region were calculated for several cases using the analytic expressions (26), (28) and (29), and were compared with the losses estimated using 2D FE simulations. Table 2 presents the eddy current power losses obtained for thirteen different configurations of the tank wall, varying the volume occupied by the stainless insert. In each case, the corresponding radial distance of the insert is shown. It can be observed that the losses calculated in all cases differ by less than $3 \%$, which confirms the correctness of the analytical expressions presented previously. Figure 6 presents also the power losses in the tank wall, calculated with the two approaches, in a graphical way. This graph shows the reduction of the total power losses in the tank wall according to the increment of the radial distance $c-a$, as expected. Although these results could point to the use of transformer tanks made exclusively with stainless steel, at least in the bushing region, this material is more expensive than the low-carbon steel, meaning a greater investment to manufacture the transformer. 


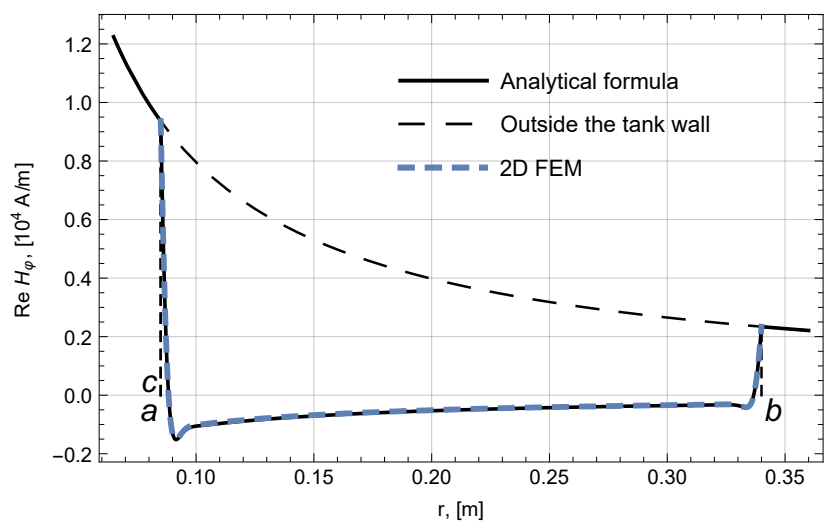

(a)

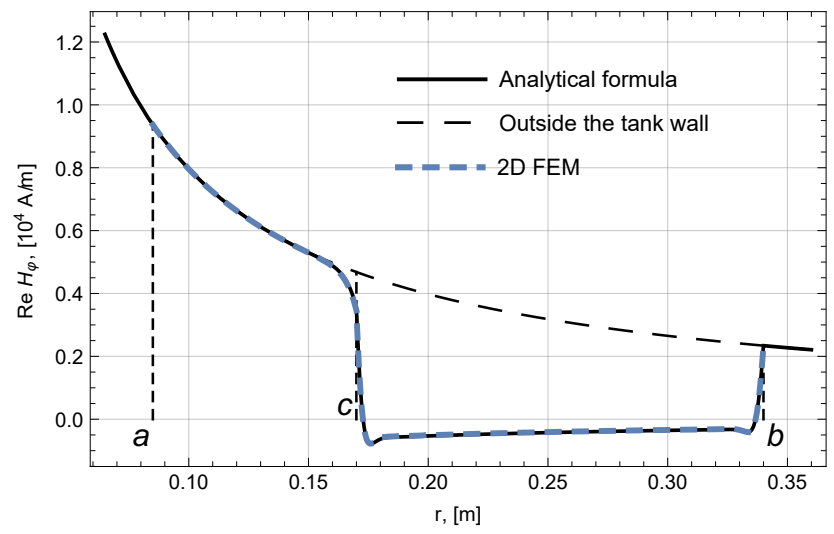

(c)

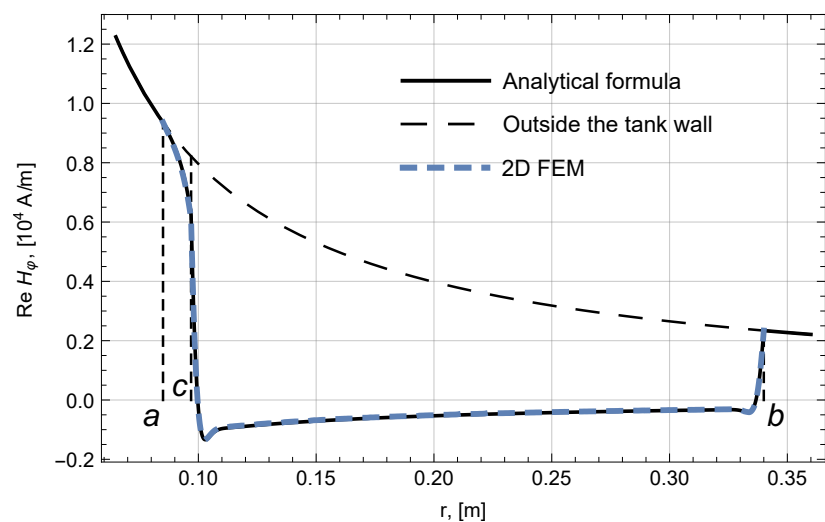

(b)

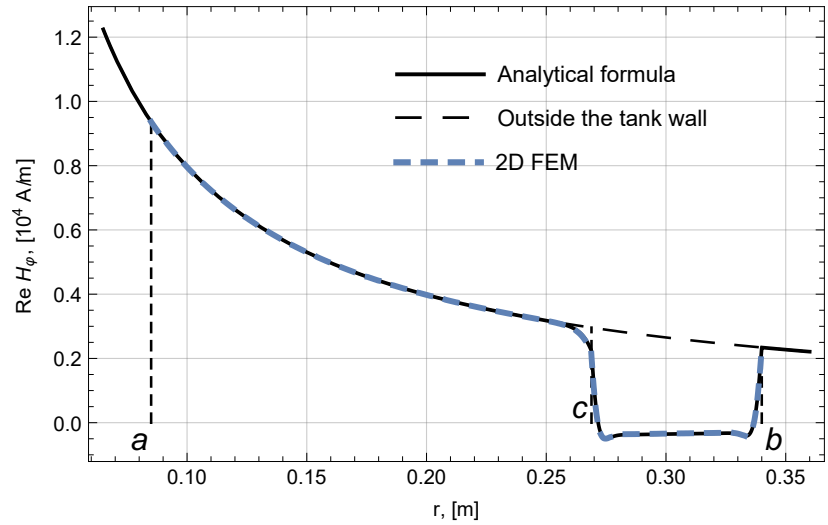

(d)

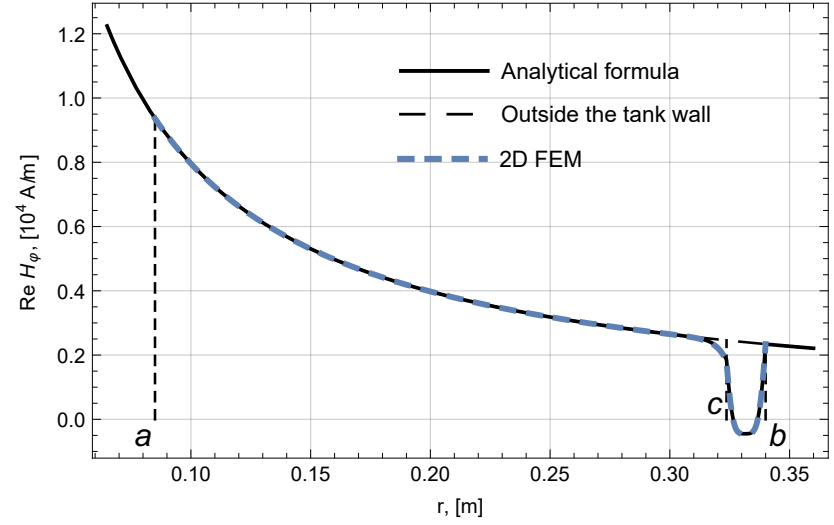

(e)

Figure 5. Magnetic field $H_{\varphi}$ evaluated at $z=0$ and $z=h / 2$. (a) Case $c=a$. (b) Case $c-a=11.914 \mathrm{~mm}$. (c) Case $c-a=85 \mathrm{~mm}$. (d) Case $c-a=183.89 \mathrm{~mm}$. (e) Case $c-a=238.67 \mathrm{~mm}$. 
Table 2. Eddy current losses in the tank wall.

\begin{tabular}{|c|c|c|c|c|c|}
\hline Case & $\begin{array}{c}\text { Insert } \\
\text { Volume } \\
(\%)\end{array}$ & $\begin{array}{l}\text { Radial Distance } \\
\text { Insert } c-a \\
\text { (mm) }\end{array}$ & $\begin{array}{c}P_{e} \\
(W) \\
\text { Analytical }\end{array}$ & $\begin{array}{c}P_{e} \\
(W) \\
\text { Numerical }\end{array}$ & $\begin{array}{c}\text { Relative } \\
\text { Error } \\
(\%)\end{array}$ \\
\hline 1 & 0 & 0 & 334.345 & 333.313 & 0.308 \\
\hline 2 & 1 & 6.152 & 317.872 & 316.813 & 0.333 \\
\hline 3 & 2 & 11.914 & 303.079 & 302.003 & 0.355 \\
\hline 4 & 5 & 27.444 & 267.309 & 266.387 & 0.344 \\
\hline 5 & 10 & 49.396 & 224.551 & 223.852 & 0.311 \\
\hline 6 & 20 & 85.000 & 168.447 & 168.018 & 0.254 \\
\hline 7 & 30 & 114.34 & 130.565 & 130.330 & 0.18 \\
\hline 8 & 40 & 139.88 & 101.941 & 101.836 & 0.103 \\
\hline 9 & 50 & 162.81 & 78.918 & 78.965 & 0.059 \\
\hline 10 & 60 & 183.89 & 59.669 & 59.799 & 0.217 \\
\hline 11 & 70 & 203.24 & 43.135 & 43.380 & 0.568 \\
\hline 12 & 80 & 221.47 & 28.630 & 28.975 & 1.205 \\
\hline 13 & 90 & 238.67 & 15.724 & 16.142 & 2.658 \\
\hline
\end{tabular}

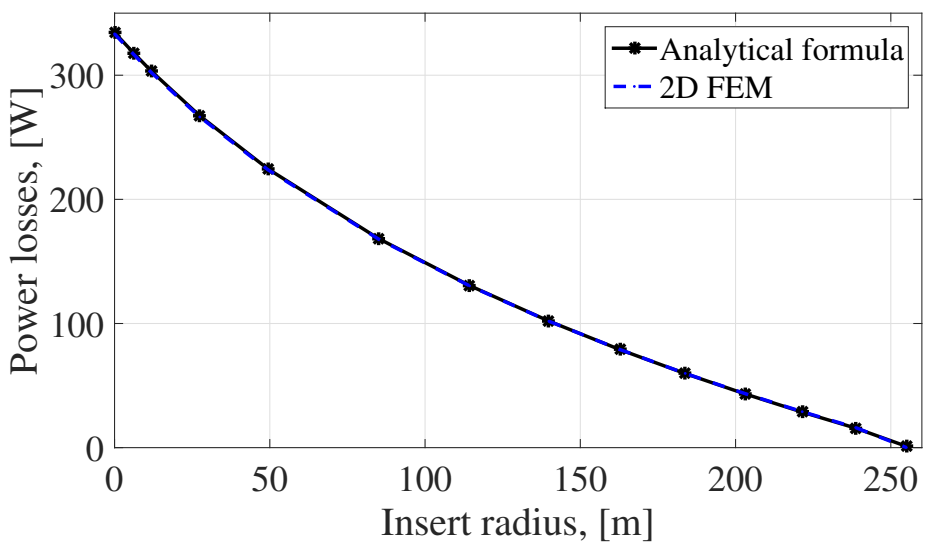

Figure 6. Power losses for different SSI dimensions.

\section{Limits and Applicability of the Analytical Solution}

Solution (24) was obtained under the assumption of axial symmetry. The edge effect on the external border was neglected by formally taking the limit: $b \rightarrow \infty$. This approach (axial symmetry) is expected to be applicable to other cases such as when the conductor crosses the covering plate not at the plate centre, but closer to the border of the transformer cover. This is due to the skin effect, making the magnetic field decay exponentially from the hole border (see Figure 7). However, in a layer near the external border, the magnetic field increases exponentially up to values of the magnetic field outside the plate (Figure 7). Therefore, if the conductor is situated too close to the border, the edge effect may become considerable.

Nevertheless, there is a case where this effect could become considerable. Just near the external border, the magnetic field increases exponentially up to values of the magnetic field outside the plate (see Figure 7). If the conductor is far away from the external border, the magnetic field quantity near the plate border is small enough so that the external border effect can be neglected. In the case of the conductor crossing the plate nearby the plate border, the edge effect becomes considerable. However, this effect is presented only in a small region shown in Figure 8, so that its contribution to the complete value of eddy current losses is small. Therefore, the only restriction of our analytical solution is $\delta_{1} \ll d$, which is normally accomplished in actual transformers. 

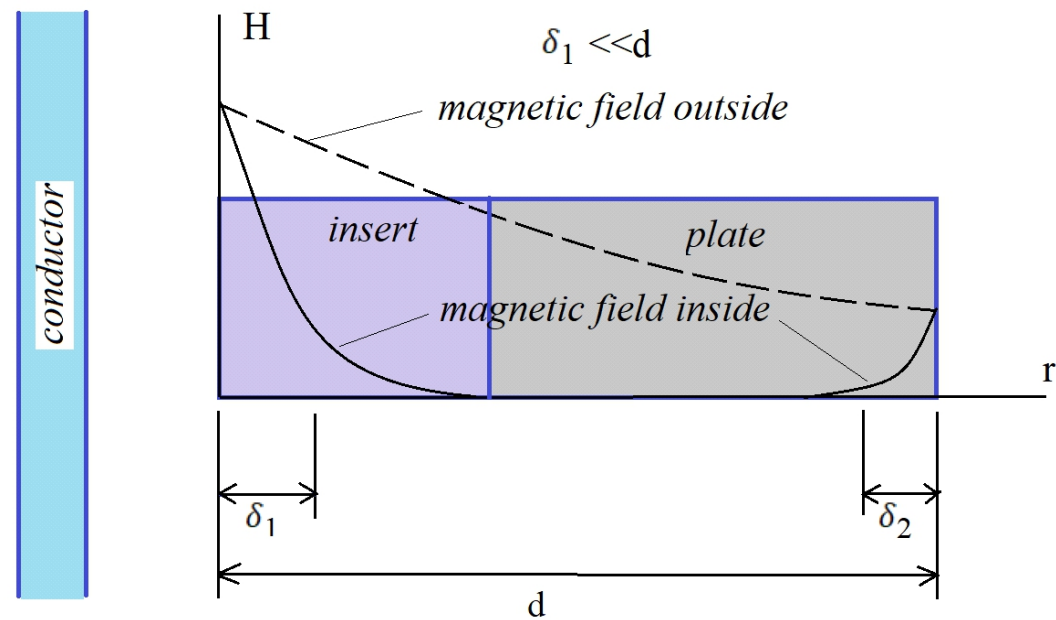

Figure 7. Magnetic field behaviour in the insert and the neighbouring plate material.

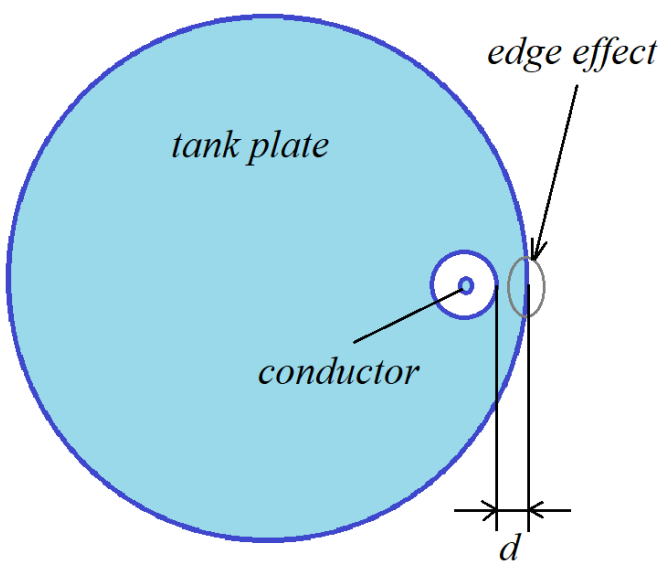

Figure 8. Limiting case of a conductor close to the plate border.

\section{Conclusions}

A new analytical model to determine the magnetic field distribution around the bushing region in the transformer tank, and considering the existence of a SSI, has been developed. The results obtained with the proposed model were validated with detailed 2D FE simulations. All the cases considered show great concordance between analytical and numerical solutions. A relative error was calculated in order to compare quantitatively the analytical model with the numerical one. This way, it was shown that the solutions differed by less than $2 \%$ in all the simulated cases. A formula to calculate the eddy current losses was also developed. The power losses were calculated for a total of thirteen different cases varying the radial distance of the insert, and again the analytical results were very close to the numerical ones. The results show that stray losses in the tank are reduced with increases in the SSI dimensions. The relative errors between stray losses, calculated with the analytical and the FE models, for all cases were less than 3\%.

These equations can serve as a basis to develop a thermal analysis in the bushing regions or an economical analysis of the insert cost against the savings due to the power loss reduction. Therefore, the new analytical model can be a useful tool for transformer designers who are interested in obtaining the optimal size of non-magnetic inserts in the tank wall, according to the rated values and dimensions of the transformer. 
Author Contributions: Conceptualization, J.C.O.-G., S.M. and M.A.C.-S.; methodology, S.M., M.A.C.S. and J.C.O.-G.; software, M.A.C.-S., E.M.-V., R.E.-P. and V.M.J.-M.; validation, S.M. and M.A.C.-S.; formal analysis, S.M. and M.A.C.-S.; investigation, S.M. and M.A.C.-S.; resources, J.C.O.-G., R.E.-P. and V.M.J.-M.; data curation, S.M. and M.A.C.-S.; writing-original draft preparation, S.M. and M.A.C.-S.; writing - review and editing, J.C.O.-G., E.M.-V. and R.E.-P.; visualization, S.M., J.C.O.-G., E.M.-V., R.E.-P. and V.M.J.-M.; supervision, S.M., J.C.O.-G., E.M.-V. and R.E.-P.; project administration, J.C.O.-G. and V.M.-J.M.; funding acquisition, J.C.O.-G., R.E.-P. and V.M.J.-M. All authors have read and agreed to the published version of the manuscript.

Funding: The authors are grateful for the financial support provided by the following CONACYT projects: CBS-2015/256519 and CB-2015/257598.

Conflicts of Interest: The authors declare no conflict of interest.

\section{References}

1. M. Del Vecchio, R.; Poulin, B.; Feghali, P.T.; Shah, D.M.; Ahuja, R. Transformer Design Principles: With Applications to Core-Form Power Transformers; CRC Press: Boca Raton, FL, USA, 2017.

2. Huerta-Rosales, J.R.; Granados-Lieberman, D.; Amezquita-Sanchez, J.P.; Camarena-Martinez, D.; Valtierra-Rodriguez, M. Vibration Signal Processing-Based Detection of Short-Circuited Turns in Transformers: A Nonlinear Mode Decomposition Approach. Mathematics 2020, 8, 575. [CrossRef]

3. Karsai, K.; Kerényi, D.; Kiss, L. Large Power Transformers; Studies in Electrical and Electronic Engineering 25; Elsevier: Amsterdam, The Netherlands, 1987.

4. Turowski, J.; Pelikant, A. Eddy Current Losses and Hot-spot Evaluation in Cover Plates of Power Transformers. EE Proc. Electr. Power Appl. 1997, 144, 435-440. [CrossRef]

5. Turowski, J. Losses in Cover Plates of single and Three Phase Power Transformers. Rozpr. Elektrotech. 1959, 1, 87-119.

6. Olivares, J.C.; Escarela-Perez, R.; Kulkarni, S.; Leon, F.D.; Melgoza-Vasquez, E.; Hernández-Anaya, O. Improved Insert Geometry for Reducing Tank-wall Losses in Pad-mounted Transformers. IEEE Trans. Power Deliv. 2004, 19, 1120-1126. [CrossRef]

7. Olivares, J.C.; Escarela-Perez, R.; Kulkarni, S.; Leon, F.D.; Venegas-Vega, M. 2D Finite-element Determination of Tank Wall Losses in Pad-mounted Transformers. Electr. Power Res. 2004, 71, 179-185. [CrossRef]

8. Kumbhar, G.B.; Mahajan, S.M.; Collett, W.L. Reduction of Loss and Local Overheating in the Tank of a Current Transformer. IEEE Trans. Power Deliv. 2010, 25, 2519-2525. [CrossRef]

9. Lopez-Fernandez, X.M.; Penabad-Duran, P.; Turowski, J. Three-Dimensional Methodology for the Overheating Hazard Assessment on Transformer Covers. IEEE Trans. Ind. Appl. 2012, 48, 1549-1555. [CrossRef]

10. Maximov, S.; Olivares-Galvan, J.C.; Escarela-Perez, R.; Magdaleno-Adame, S.; Campero-Littlewood, E. New Analytical Formulae for Electromagnetic Field and Eddy Current Losses in Bushing Regions of Transformers. IEEE Trans. Magn. 2015, 51, 6300710. [CrossRef]

11. Penabad-Durán, P.; Barba, P.D.; Lopez-Fernandez, X.; Turowski, J. Electromagnetic and Thermal Parameter Identification Method for Best Prediction of Temperature Distribution on Transformer Tank Covers. COMPEL Int. J. Comput. Math. Electr. Electron. Eng. 2015, 34, 485-495. [CrossRef]

12. Zahedi, M.Z.; Iskender, I. Evaluation of Eddy Current Losses in the Cover Plates of Distribution Transformers. Int. J. Tech. Phys. Probl. Eng. 2018, 10, 27-33.

13. Zia Zahedi, M.; Iskender, I. Nonlinear Adaptive Magneto-Thermal Analysis at Bushing Regions of a Transformers Cover Using Finite Difference Method. J. Therm. Sci. Eng. Appl. 2018, 11. [CrossRef]

14. Khan, S.; Maximov, S.; Escarela-Perez, R.; Olivares-Galvan, J.C.; Melgoza-Vazquez, E.; Lopez-Garcia, I. Computation of Stray Losses in Transformer Bushing Regions Considering Harmonics in the Load Current. Appl. Sci. 2020, 10, 3527. [CrossRef]

15. De Oliveira, L.F.; Sadowski, N.; Cabral, S.H.L. Alternative Model for Computing Transformer Tank Induced Losses in the Time Domain. IET Electr. Power Appl. 2020. [CrossRef]

16. Lanczos, C. Applied Analysis. Phys. Today 10 1957, 6, 44. [CrossRef]

17. Wilcox, D.J.; Conlon, M.; Hurley, W.G. Calculation of Self and Mutual Impedance for Coils on Ferromagnetic Cores. IEE Proc. 1988, 135, 470-476. [CrossRef]

18. Corona-Sánchez, M.A.; Melgoza-Vázquez, E.; Maximov, S.; Escarela-Perez, R. An Improved Time-Harmonic 2-D Eddy Current Finite-Element H Formulation. IEEE Trans. Magn. 2017, 53, 1-4. [CrossRef]

19. Melgoza, E.; Escarela-Pérez, R.; Corona-Sánchez, M.A. Applications of Computational Electromagnetism in Electric Power Engineering. In Proceedings of the 2016 IEEE PES Transmission Distribution Conference and Exposition-Latin America (PES T\& D-LA), Morelia, Mexico, 20-24 September 2016; pp. 1-6. 